\title{
A fluorescent thermometer operating in aggregation-induced emission mechanism: probing thermal transitions of PNIPAM in water $\dagger$
}

\author{
Li Tang, ${ }^{a}$ Jia Ke Jin, ${ }^{a}$ Anjun Qin, ${ }^{a}$ Wang Zhang Yuan, ${ }^{a b}$ Yu Mao, ${ }^{a}$ Ju Mei, ${ }^{a}$ Jing Zhi Sun $* a$ \\ and Ben Zhong Tang*ab
}

Received (in Cambridge, UK) 14th April 2009, Accepted 18th June 2009

First published as an Advance Article on the web 10th July 2009

DOI: $10.1039 / b 907382 \mathrm{e}$

\begin{abstract}
$\operatorname{Poly}(N$-isopropylacrylamide) was labelled using a fluorogen with an aggregation-induced emission feature by direct polymerization; the label served as a fluorogenic probe that reveals fine details in the thermal transitions in the aqueous solution of the polymer; the working mode was readily tuned between non-monotonic and monotonic by changing the labelling degree of the polymer.
\end{abstract}

Luminogenic probes have attracted much interest due to their ultrahigh sensitivity and superfast response. Thanks to the enthusiastic effort of scientists working in the area, a large variety of fluorescence (FL) sensors for the analyses of chemical species and physical processes have been developed. ${ }^{1}$ Sensitive and fast monitoring of temperature change is of great importance to the development of biosensors, as biological events are often accompanied with a variation in body temperature. ${ }^{2}$ Hyperpyrexia, for example, is a high fever. At high temperature, biopolymers undergo conformation changes, leading to protein aggregation (fibrillogenesis) and fatal implications such as muscle rigidification and brain death. ${ }^{3}$

Poly $(N$-isopropylacrylamide) (PNIPAM) is a thermoresponsive synthetic polymer that undergoes the conformational transition from hydrated coil to dehydrated globule in water at a temperature close to that of the human body. The polymer is thus a nice synthetic model for investigating thermal transitions in natural polymer systems. A reversible liquid-solid phase transition occurs in an aqueous mixture of PNIPAM at around $32{ }^{\circ} \mathrm{C}$, which is defined as its lower critical solution temperature (LCST). ${ }^{4}$ The biocompatibility of PNIPAM and its near-body-temperature LCST have stimulated much work on the exploration of its utilization in biotechnology, especially in the development of temperature-responsive drug delivery systems. ${ }^{5}$ The investigation of thermal transitions of PNIPAM is thus of great technological value in its own right.

Fluorophores have been incorporated into PNIPAM structure for the purpose of studying their thermal transitions using the FL technique. ${ }^{6-8}$ Pyrene and rhodamine, for

\footnotetext{
${ }^{a}$ Institute of Biomedical Macromolecules, Department of Polymer Science \& Engineering, Zhejiang University, Hangzhou, China. E-mail: sunjz@zju.edu.cn; Fax: +8657187953734

${ }^{b}$ Department of Chemistry, The Hong Kong University of Science \& Technology, Clear Water Bay, Kowloon, Hong Kong, China. E-mail:tangbenz@ust.hk; Fax:+852 23581594

$\dagger$ Electronic supplementary information (ESI) available: Text, schemes and table describing experimental procedures and characterisation data for the syntheses of $\mathbf{1}$ and $\mathbf{P 1}$; figures showing the NMR, UV and FL spectra and turbidity data for $\mathbf{P 1}$ at various concentrations and temperatures and the calibration curve for determining the degrees of labelling in P1a-P1c. See DOI: 10.1039/b907382e
}

example, were attached to a copolymer of NIPAM and $N$-(acryloxy)succinimide via a polymer reaction. ${ }^{7,8}$ In the pyrene system, the ratio of its excimer-monomer emissions varied with temperature, ${ }^{7}$ while in the rhodamine system, its FL changed with temperature due to dehydration of PNIPAM at high temperatures under acidic conditions. ${ }^{8}$ In this work, we explored the possibility of finding a direct way for labelling PNIPAM and developing an FL thermometer working in a new mechanism and operating under neutral conditions.

We have recently observed a new phenomenon of aggregationinduced emission (AIE). ${ }^{9,10}$ Tetraphenylethene (TPE) is a typical AIE dye, which is non-emissive when dissolved in solvents in which it has high solubility but become highly emissive when aggregated in solvents in which it is poorly soluble. ${ }^{9}$ Its AIE effect is rationalized to be caused by restricted intramolecular rotation (RIR) of its phenyl peripheries against the central double bond in the aggregate state. ${ }^{10}$ Water is a good and poor solvent for PNIPAM below and above its LCST, respectively. It is envisaged that when a PNIPAM chain is labelled by a TPE fluorogen, the thermally induced chain aggregation may alter the emission of the TPE label, thus enabling it to function as an FL thermometer for monitoring thermal transitions of PNIPAM in water.

According to the design principle elaborated above, the TPE fluorogen was functionalized with a vinylidene group using a copper-catalyzed click reaction (Scheme 1). ${ }^{11}$ The TPE derivative was incorporated into the PNIPAM structure by radical polymerization of the NIPAM monomer in the presence of 1 . Since the LCST of PNIPAM is sensitive to structural modifications, ${ }^{4}$ small amounts of $\mathbf{1}(\sim 0.3-1 \%)$ were used in the polymerization reactions, in an effort to minimize the structural perturbation to the polymer properties.

The resultant polymers (P1) were carefully purified and characterized (see ESI $\uparrow$ for details). Their GPC data indicate

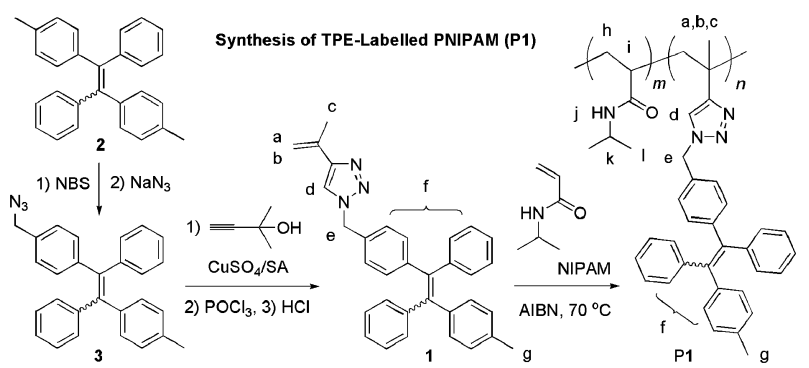

Scheme 1 Preparations of TPE derivative 1 and TPE-labelled PNIPAM P1. For P1a $m: n=372: 1$; for P1b $m: n=171: 1$; for P1c $m: n=89: 1$. 
that the incorporation of $\mathbf{1}$ into the PNIPAM structure has exerted little effect on the molecular weights and polydispersity indexes (ESI $\dagger$, Table S1). In the ${ }^{1} \mathrm{H}$ NMR spectra of P1, the protons of the aromatic rings resonate at $\delta \sim 7.0-7.8$ (ESI $\dagger$, Fig. S1-S3), confirming that the PNIPAM chain has indeed been labelled by the TPE fluorogen by the simple procedure of direct polymerization. Using the calibration curve drawn from the UV absorption data (ESI $\dagger$, Fig. S4 and S5), the degrees of labelling in P1a, P1b and P1c are determined to be $0.27 \%$, $0.58 \%$ and $1.12 \%$, respectively (ESI $\dagger$, Table $\mathrm{S} 1$ ).

The polymer with the lowest degree of labelling (P1a) is nonemissive when dissolved in THF, a good solvent for both TPE and PNIPAM (Fig. 1). The FL becomes visible when large amounts ( $\geq 70 \%$ ) of water, a poor solvent for TPE, are added into the THF solution of P1a, indicating that the TPE label maintains its AIE activity after being incorporated into the PNIPAM structure. The polymer with the highest degree of labelling (P1c) shows similar AIE behaviour (ESI $\dagger$, Fig. S6). The FL intensity of P1 is increased with increasing extent of labelling (ESI $\dagger$, Fig. S7) and concentration of polymer (ESI $\dagger$, Fig. S8) $\dagger$ This serves as another proof for the AIE nature of the TPE label, because the emission of a "conventional" fluorophore is normally weakened, rather than strengthened, with increasing concentration.

The temperature effect on the FL behaviour of P1a in water is shown in Fig. 2A. Little change in the emission intensity of P1a is recorded when its aqueous solution is heated from 14 to $25{ }^{\circ} \mathrm{C}$, while a small bump is observed in the temperature region of 25 to $29{ }^{\circ} \mathrm{C}$. Above $29{ }^{\circ} \mathrm{C}$, the FL intensity of P1a swiftly increases with increasing temperature and reaches a maximum at $34.2{ }^{\circ} \mathrm{C}$. Further heating leads to a continuing decrease in the FL intensity. When the aqueous mixture of P1a is cooled from 50 to $18{ }^{\circ} \mathrm{C}$, a largely reversible FL intensitytemperature curve is obtained, with the big peak and small bump recorded in the similar temperature regions. When the polymer concentration is changed from 1 to $0.1 \mathrm{mg} \mathrm{mL}^{-1}$, the starting point for the FL enhancement is increased from $\sim 25$ to $\sim 32{ }^{\circ} \mathrm{C}$ and the $\mathrm{FL}$ maximum is shifted from $\sim 34$ to $\sim 37{ }^{\circ} \mathrm{C}$ (ESI $\dagger$, Fig. S9). This indicates that the

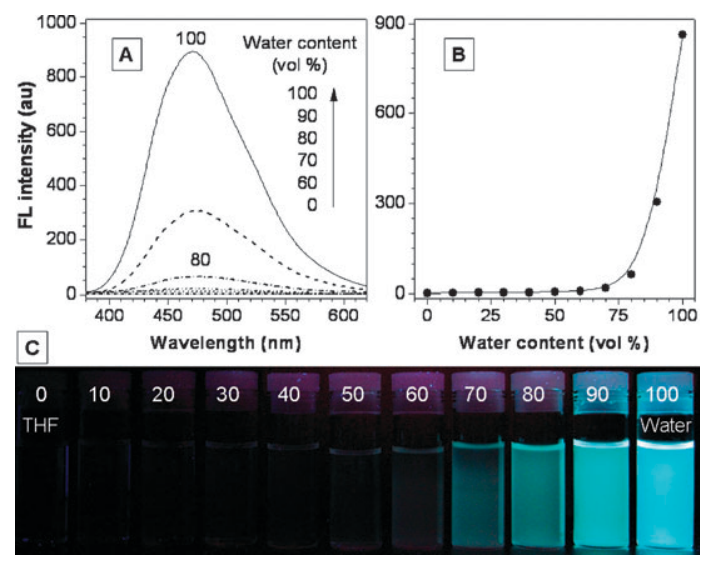

Fig. 1 (A) FL spectra of P1a in water-THF mixtures with different water contents measured at $17^{\circ} \mathrm{C}\left(\lambda_{\mathrm{ex}}=322 \mathrm{~nm},[\mathbf{P 1 a}]=1 \mathrm{mg} \mathrm{mL}^{-1}\right)$. (B) Change of FL maximum of P1a with water content of the aqueous mixture. (C) Photographs of P1a solutions taken under UV illumination. thermal transitions of the polymer are tunable by such simple external manipulations ${ }^{4}$ as changing its solution concentration.

The FL of P1a is temperature dependant but its thermal response is non-monotonic, which is more informative than the monotonic pattern afforded by other analytical techniques. ${ }^{4}$ Dynamic light scattering (DLS) and solution turbidity measurements, for example, furnish curves that start to monotonously increase from 28 and $31{ }^{\circ} \mathrm{C}$, respectively (Fig. 2B). These data imply that the PNIPAM chains start to aggregate from the specific temperatures but fail to offer detailed information about the transition processes because of their lower sensitivity, in comparison to that of the FL technique.

To collect more information about chain conformation of P1a and to gain more insight into its thermal transitions, its ${ }^{1} \mathrm{H}$ NMR spectra are measured in different solvents at various temperatures. The NMR spectrum taken at room temperature in DMSO, a good solvent for both PNIPAM and TPE, contains resonance signals of all the protons, as marked in Fig. 3A. In water, however, the peaks for the aromatic protons in the downfield disappear. As water is a polar solvent, the hydrophobic TPE labels may have been wrapped by the polymer coils or aggregated into tiny clusters. The wrapping and aggregation must have been loose, because the P1a solution is homogeneous and transparent. The loose wrapping and aggregation partially hamper intramolecular rotation of the aryl rings of the TPE label, which explains why P1a is luminescent in water ( $c f$., Fig. 1).

When P1a solution is heated to $25^{\circ} \mathrm{C}$, the PNIPAM chains start to dehydrate, probably from the isopropyl pendants. ${ }^{4}$ This partially breaks the water cages surrounding the polymer coils and promotes the dehydrated chain segments to undergo a coil-globule transition. The change at this initial stage is too small to be detected by DLS, UV and NMR analyses. The volume shrinkage accompanying the coil-globule transition, although small at this stage, is picked up by the TPE label. The reduced volume impedes its intramolecular rotation, thus making it more emissive ( $c f$., Fig. 2A).

In the temperature region of 29 to $34{ }^{\circ} \mathrm{C}$, coil-globule transition becomes active. At this stage, not just the pendants and segments but the whole polymer chains dehydrate. The dehydration results in the formation of compact aggregates, as evidenced by the decrease in the NMR peak intensities (Fig. 3D). This greatly activates the RIR process of the TPE label. The fluorogen thus becomes highly emissive, a manifestation of its AIE effect.

Further heating of the polymer mixture to above $34{ }^{\circ} \mathrm{C}$ may cause little change in the compactness of the polymer aggregates because the phase transition has already finished at the LCST, although the aggregates continue to grow in size. Indeed, the aggregates become so big at the high temperature that NMR spectra cannot be taken (Fig. 3E). The TPE labels buried in the big aggregates can hardly be reached by the UV excitation beam. Meanwhile the high-temperature heating activates the molecular motions such as intramolecular rotation and vibration of the TPE fluorogen. These two effects collectively make the polymer mixture less emissive, as can be seen from the continuous decrease in the FL intensity in the high temperature region ( $c f$. , Fig. 2A). 


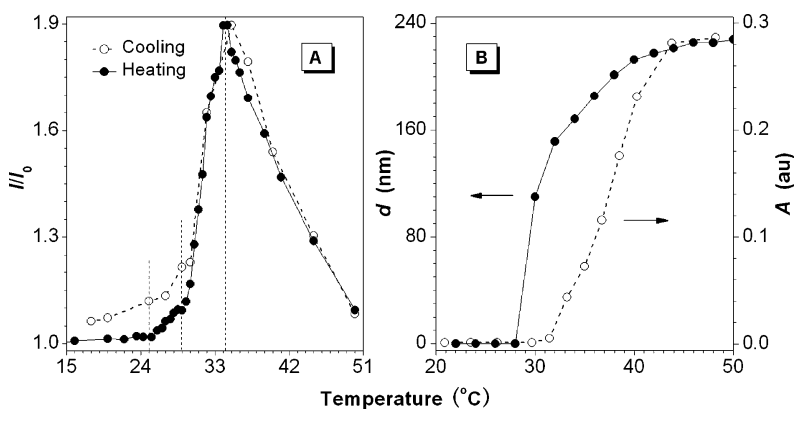

Fig. 2 Effects of temperature on (A) FL intensity (I) at $468 \mathrm{~nm}$ and (B) particle size $(d)$ and solution turbidity of P1a $\left(1 \mathrm{mg} \mathrm{mL}^{-1}\right)$. $\lambda_{\text {ex }}=322 \mathrm{~nm} ; I_{0}=$ intensity at $468 \mathrm{~nm}$ at $14{ }^{\circ} \mathrm{C}$. Turbidity data measured as a function of absorbance $(A)$ at $650 \mathrm{~nm}$.

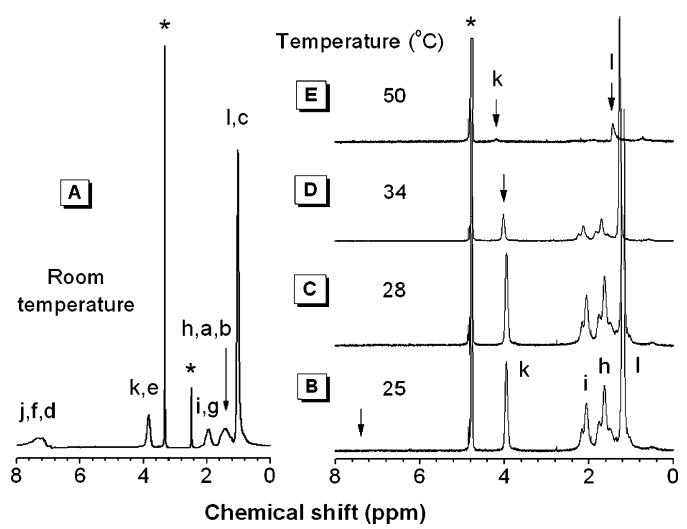

Fig. $3{ }^{1} \mathrm{H}$ NMR spectra of P1a taken (A) in DMSO- $d_{6}$ at $\sim 23{ }^{\circ} \mathrm{C}$ (room temperature) and (B-E) in $\mathrm{D}_{2} \mathrm{O}$ at various temperatures. The labels for the resonance peaks correspond to those given in Scheme 1 and the solvent and water peaks are marked with asterisks.

Surprisingly, the PNIPAM with the higher TPE content, i.e., P1b, behaves very differently from P1a. Its FL intensity monotonously decreases with increasing temperature, although a slope change is recorded at $28{ }^{\circ} \mathrm{C}$ (Fig. 4A). The chain conformation of PNIPAM is known to be extremely sensitive to its molecular structure: even a subtle change in the end group introduced by an initiator fragment can cause a big change. ${ }^{4}$ The hydrophobic interaction between the TPE labels in P1b may have enabled the fluorogens to aggregate, even at room temperature. These nanoclusters may serve as nuclei to attract hydrophobic backbones of PNIPAM chains to conglobulate. As a support to this conjecture, the water solution of P1b is more turbid (Fig. 4B) and more emissive (Fig. 4A) than that of P1a at room temperature.

Heating a water solution of $\mathbf{P 1}$ generally exerts two antagonistic effects on the TEP label. The thermally induced conglobulation of the PNIPAM chains suppresses, whereas the thermally activated molecular motions encourages, the intramolecular rotation in the fluorogen. The former and latter effects strengthen and weaken the emission of the fluorogen, respectively. Since the TPE labels in P1b have already aggregated in the water solution at room temperature, heating would predominantly exert the latter effect on the fluorogen, leading to the continuous decrease in the FL intensity. The similar behaviour shown by P1c verifies that it is the "large" amount of the TPE labels in the polymer that has changed the

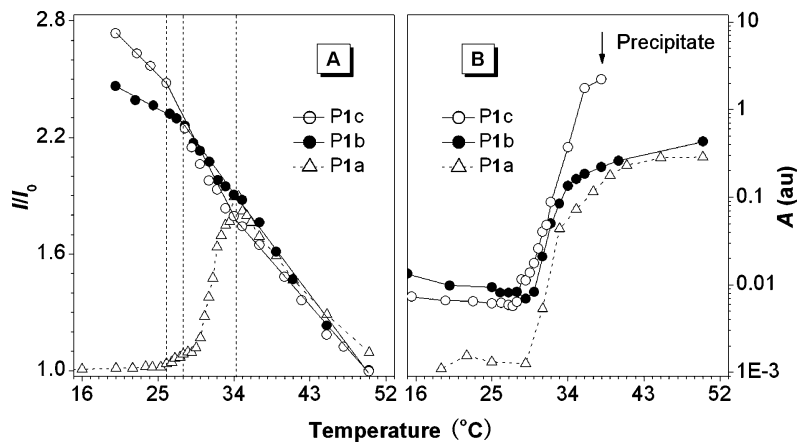

Fig. 4 Effects of temperature on (A) FL intensity (I) at $468 \mathrm{~nm}$ and (B) particle size $(d)$ and solution turbidity of $\mathbf{P 1}\left(1 \mathrm{mg} \mathrm{mL}^{-1}\right)$. $\lambda_{\mathrm{ex}}=322 \mathrm{~nm} ; I_{0}=\mathrm{FL}$ intensity at $468 \mathrm{~nm}$ at $14^{\circ} \mathrm{C}$. Turbidity data measured as a function of absorbance $(A)$ at $650 \mathrm{~nm}$.

working mode of the FL thermometer from non-monotonic to monotonic tone.

In summary, an FL thermometer system working in a novel AIE mechanism under neutral conditions has been developed in this work by labelling a PNIPAM chain with TPE fluorogen (1) through a simple polymerization reaction. The PNIPAM with a small number of TPE labels (P1a) works as a non-monotonic probe that reveals delicate details about the thermal transitions of PNIPAM, which are inaccessible by other analytical techniques. The working tone is tuned by simply changing the labelling degree: the polymers labelled with large amounts of TPE fluorogens (P1b and P1c) work in a monotonic mode, due to the prevailing effect of the thermally activated intramolecular rotation over that of the RIR process in the polymer systems.

This project was supported by the National Science Foundation of China (50873086), the Ministry of Science \& Technology of China (2009CB623605), and the Hong Kong Research Grants Council (603008). B.Z.T. thanks the support from the Cao Guangbiao Foundation of Zhejiang University.

\section{Notes and references}

1 For reviews, see: (a) S. W. Thomas III, G. D. Joly and T. M. Swager, Chem. Rev., 2007, 107, 1339; (b) D. W. Domaille, E. L. Que and C. J. Chang, Nat. Chem. Biol., 2008, 4, 168.

2 C. Gota, K. Okabe, T. Funatsu, Y. Harada and S. Uchiyamam, J. Am. Chem. Soc., 2009, 131, 2766.

3 For a review, see: J. McLaurin, D. Yang, C. M. Yip and P. E. Fraser, J. Struct. Biol., 2000, 130, 259.

4 (a) H. Inomata, S. Goto and S. Saito, Macromolecules, 1990, 23, 4887; (b) H. G. Schild, Prog. Polym. Sci., 1992, 17, 163; (c) G. Zhang and C. Wu, Adv. Polym. Sci., 2006, 195, 101; (d) Z. M. O. Rzaev, S. Dincer and E. Piskin, Prog. Polym. Sci., 2007, 32, 534.

5 S. Chaterji, I. K. Kwon and K. Park, Prog. Polym. Sci., 2007, 32, 1083.

6 H. Ringsdorf, J. Simon and F. M. Winnik, ACS Symp. Ser., 1993, 532, 216.

7 F. M. Winnik, Macromolecules, 1990, 23, 233.

8 Y. Shiraishi, R. Miyamoto, X. Zhang and T. Hirai, Org. Lett., 2007, 9, 3921.

9 J. Luo, Z. Xie, J. W. Y. Lam, L. Cheng, H. Chen, C. Qiu, H. Kwok, X. Zhan, Y. Liu, D. Zhu and B. Z. Tang, Chem. Commun., 2001, 1740.

10 Y. Hong, J. W. Y. Lam and B. Z. Tang, Chem. Commun., 2009, 4332.

11 A. Qin, L. Tang, J. W. Y. Lam, C. K. W. Jim, Y. Yu, H. Zhao, J. Sun and B. Z. Tang, Adv. Funct. Mater., 2009, 19, 1891. 